\title{
LPV Control Synthesis for Sensor Senescence in Feedback Control System
}

\author{
AQEEL MADHAG
}

\begin{tabular}{l}
\hline \hline Article Info \\
\hline Article history: \\
Received Oct 10, 2020 \\
Revised May 10, 2021 \\
Accepted May 17, 2021 \\
\hline
\end{tabular}

\section{Keywords:}

Discrete-Time (DT)

Gain Scheduled (GS)

Linear Parameter-Varying (LPV)

system

Dynamic Output Feedback

(DOF).

\begin{abstract}
The senescence of system sensor(s) probably causing degenerate system behavior or system breakdown. Motivated by that, this work considers the synthesis of a gain scheduling control for linear parameter varying (LPV) system via linear matrix inequalities (LMIs) techniques such that sensors senescence information incorporated in the design of the controller. That is, the degradation of sensor effectiveness due to senescence is modeled by the variation of sensor measurements' noise co-variance. The sensors' senescence information is incorporated as a part of the scheduling parameters for the LPV controller. The synthesis control matrices via linear matrix inequalities have been re-structured and re-formalized in a way that they incorporate the sensors senescence information to synthesize a gain scheduling dynamic output feedback (GS - DOF) control. That is, the existing GS - DOF control design LMIs have been modified to include the noise co-variance matrix. The significant achievement is the control design conditions description of GS - DOF control in a way that ensures the desired integrated $\mathrm{H}_{2} \& \mathrm{H}_{\infty}$ performances in the presence of sensors senescence, where sensors' senescence information is incorporated as a part of the scheduling parameters for the LPV controller. In addition, two sets of controllers have been synthesized and studied. The simulation expresses the benefits of the proposed controllers, and closed loop system $\mathrm{H}_{\infty}$ and $\mathrm{H}_{2}$ performances are also studied. The synthesized controller ensures the performance associate with a closed loop, the closed loop system stability, and the scheme of control is simple enough for real time implementations.
\end{abstract}

\section{Corresponding Author:}

AQEEL MADHAG,

Al-Furat Al-Awsat Technical University,

Al-Najaf Technical Engineering College,

Kufa, Najaf Governorate 00964, IRAQ.

Ph.D. Electrical and Computer Engineering,

4140 Engineering Building, Michigan State University,

East Lansing, MI 48824-1226, U.S.A.

Email: asr19802007@gmail.com (or madhagaq@atu.edu.iq)

ORCID: 0000-0002-7731-0272

Research ID: N-3948-2018

\section{INTRODUCTION}

Dynamical systems in the control community are represented in a framework such that the available techniques and software can handle the control formalization. The linear time-invariant (LTI) or linear time-varying (LTV) modeling of the dynamical system has been widely used in control literature. The point against them is that all system matrices are linear, which is conservative formalization. Nonlinear modeling of a dynamical system is much better than the linear one, but the lack of simplicity and available parsers to simulate it is the main difficulty. The LPV systems framework consider dynamical systems, which have been described by linear differential equations modeled data depend on time-varying parameters; that is, for the linear and nonlinear dynamics, the LPV scheme lies in-between [1]. Considering the control design problem, the goal of the LPV control synthesis issue is to design a compensator such that the system satisfies the desired performance and stability over the domain of the considered parameters. In other words, the LPV controller invests the online values of the time-varying parameters to satisfy the stability and performance over the robust controllers, where the time-varying parameters are defining the operating point of the system. That is, LPV controllers have an advantage over the classical gain scheduling controllers such that the controller is automatically gain scheduled without any ad hoc process. The point of using LPV control synthesis techniques is that they provide a structured synthesis procedure for gain scheduling controllers while allowing performance, robustness, and bandwidth restrictions to be incorporated into a coherent framework investing the available parsers in solving the LMls set of the nonlinear system. Many kinds of 
literature have investigated the LPV control synthesis since the first presenting of gain scheduling control by Ref. [2], where the focus was on the synthesis framework to ensure system stability. The other trend was for satisfying system performance, which has been considered by many researchers, for instance, Refs. [3-5]. In the same manner, the multiobjective (i.e., mixed $\mathrm{H}_{2} \& \mathrm{H}_{\infty}$ ) LPV control synthesis trend has been considered also, for example, Refs. [6-8]. The point against all the above literature is that they consider the output measurement may (or not) be affected by a known and timeinvariant statistical characteristics noise. To be specific, no sensor performance degradation due to sensor senescence had been considered, , which is the focus of this work.

Indeed, system performance is directly influenced by a sensor failure, which could lead to negative consequences. A faulty sensor (or sensors) may cause system performance degradation, system shut down, and/or fatal accident. The recent literature trend towards the effect of sensors malfunctions to the system performance in different applications, like, wind power generation [9], transmission lines [10, 11], wireless sensor networks [12, 13], and high-voltage systems [14]. The scope of fault tolerant control is not recent in literature. The common trend in recent literature is to model the sensor fault as an additive or multiplicative term (i.e., fixed value and time-invariant) in the measurement equation and augment the fault terms with system states. A virtual sensor approach was invested to cover the faulty sensor measurement(s) and ensures the nominal system performance; see Refs. [15-17]. The point missed by the literature trend mentioned above is that sensor performance degradation is a dynamical process, and model it as a fixed and time-invariant term is a conservative assumption and far away from reality and the practical world, which motivates this paper.

In this work, the faulty sensor (or sensor failure) is considered as a dynamical process (i.e., a time-varying sensor noise statistics model the sensor senescence), which affects system output performance directly and could lead to catastrophic consequences. That is, sensor fault could be caused by gradual degradation of sensor performance [18, 19], or abrupt (intermittent) performance change [20, 19], and it impacts system performance, stability, and reliability. Then, sensor performance deterioration information needs to be utilized by the closed loop control strategy in real time. Motivated by that, this work considers the utilization of the sensor effectiveness deterioration information by the closed loop control strategy in real time to reduce system breakdowns and preserves the stability for acceptable performance, where the sensor faults are due to senescence.

Consequently, the main purpose here is to synthesize multi-objective GS - DOF control preserves the desired performances in the presence of sensor effectiveness deterioration resulting from sensor senescence for an LPV (LPV) system. The GS - DOF control problem has been considered by recent literature, like, Refs. [21-27], which does not consider sensor performance deterioration. In contrast to the existing literature, this work considers sensor fault as a dynamical process (i.e., the time-varying sensor noise statistics model the sensor senescence). Specifically, the time-varying sensor measurements noise co-variance has been used to model the sensor performance deterioration due to senescence. Besides, sensor senescence information has been considered as a part of the scheduling parameters. Moreover, the control design LMIs set has been modified to incorporate the sensor noise co-variance matrix. Furthermore, the designed controller aims to ensure stability and satisfy the system's desired performances, while the sensor performance deterioration is derived by sensor senescence.

This work has many contributions. First, an integrated $\mathrm{H}_{2} \& \mathrm{H}_{\infty} \mathrm{GS}-$ DOF control problem for the discrete-time linear parameter varying (DT - LPV) system with sensors senescence is proposed. Note that the point of using LPV techniques is that they provide a structured synthesis procedure for gain scheduled controllers while allowing performance, robustness, and bandwidth restrictions to be incorporated into a coherent framework investing the available parsers in solving the LMIs set of the nonlinear system. Second, a parameterized LMIs set formulated in a way such that the sensor senescence information is invested. Third, the parameterized LMIs are employed to design a controller with scheduling parameters involved sensor senescence information to minimize the control exertion. In addition, the sensor effectiveness deterioration owing to sensor senescence is simulated by noise co-variance alteration of sensor measurements. Practically, as the sensor gets more matured, its measurement becomes more noisy, then noise co-variance alteration of sensor measurements simulates that issue. That is, sensor performance deterioration owing to sensor senescence is simulated by noise co-variance alteration of sensor measurements. Moreover, a collection of the estimated noise co-variance and time-varying parameters are invested in scheduling the controls' gains. Furthermore, two sets of controllers have been synthesized and studied. Simulation studies legitimate those controllers for a DT - LPV system. Additionally, the synthesized controllers improve system performance, $\mathrm{H}_{2}$-norm, and ensures the closed loop stability, exposed to $\mathrm{H}_{\infty}$-performance constraint. Similarly, the feasibility for real time implementations handled by the designed controllers. This paper used standard notations. The paper is laid out as following. Section 2. expresses the proposed problem, and mathematical background. Section 3. shows the design of parameterized LMI constraints for the considered integrated $\mathrm{H}_{2} \& \mathrm{H}_{\infty}$ control issue. Section 4. exhibits the simulations. Section 5. lists the conclusions. 


\section{DYNAMICAL SYSTEM PERFORMANCES}

The polytopic DT - LPV system can be described by a state-space model below,

$$
\left\{\begin{aligned}
\mathrm{x}(k+1) & =\mathcal{A}(\xi(k)) \mathrm{x}(k)+\mathcal{B}_{u}(\xi(k)) \mathrm{u}(k)+\mathcal{B}_{\infty w}(\xi(k)) w_{\infty}(k)+\mathcal{B}_{2 w}(\xi(k)) w_{2}(k), \\
\mathrm{z}_{\infty}(k) & =\mathcal{C}_{\infty z}(\xi(k)) \mathrm{x}(k)+\mathcal{D}_{\infty u}(\xi(k)) \mathrm{u}(k)+\mathcal{D}_{\infty w}(\xi(k)) w_{\infty}(k), \\
\mathrm{z}_{2}(k) & =\mathcal{C}_{2 z}(\xi(k)) \mathrm{x}(k)+\mathcal{D}_{2 u}(\xi(k)) \mathrm{u}(k), \\
\mathrm{y}(k) & =\mathcal{C}_{y}(\xi(k)) \mathrm{x}(k)+\mathcal{D}_{y v} \widetilde{v}(k),
\end{aligned}\right.
$$

where $\mathrm{z}_{2}(k) \in \mathbb{R}^{p_{2}}$ is the performance output vector; $\mathrm{z}_{\infty}(k) \in \mathbb{R}^{p_{\infty}}$ is the controlled output vector, which related to modeling error; and the other vectors and matrices are with suitable dimensions. The parameter-independent proposition is considered for matrix $\mathcal{D}_{y v}$ since the sensor noise statistics are not known in advance and they are estimated online; see Refs. $[28,18,20]$ for more details. The matrices for system (1) are proposed to possess polytopic parametrization dependent on parameters $\xi(k)=\left[\xi_{1}(k), \cdots, \xi_{n_{\xi}}(k)\right]^{T}$, which are polytopic, that is,

$$
\boldsymbol{\Omega}(\xi(k))=\sum_{r=1}^{n_{\xi}} \xi_{r}(k) \Omega_{r},
$$

where the $r^{t h}$ component of the vector $\xi(k)=\left[\xi_{1}(k), \cdots, \xi_{n_{\xi}}(k)\right]^{T}$ is $\xi_{r}(k)$; the parameter dependent matrix of system (1) is $\boldsymbol{\Omega}(\xi(k))$; and $\Omega_{r}$ is a constant matrix. The controllability and observability are proposed for system (1), where this proposition is used widely due to the non-well-defined detectability and stabilizability in the LPV control publications. The modeling error disturbance is designated by $w_{\infty}(k) \in \mathbb{R}^{r_{\infty w}}$; the process noise by $w_{2}(k) \in \mathbb{R}^{r_{2 w}}$; and the measurement noise by $\widetilde{v}(k) \in \mathbb{R}^{r} \tilde{v}$, where, the uncorrelated Gaussian white proposition is considered with their means and co-variances defined by

$$
\left\{\begin{aligned}
\mathbb{E}\left[w_{2}(k)\right] & =0, \quad \mathbb{E}\left[w_{2}(k) w_{2}^{T}(j)\right]=\mathcal{W}_{k_{2}} \delta\left(t_{k}-t_{j}\right), \forall k, j \in \mathbb{R}, \\
\mathbb{E}\left[w_{\infty}(k)\right] & =0, \quad \mathbb{E}\left[w_{\infty}(k) w_{\infty}^{T}(j)\right]=\mathcal{W}_{k_{\infty}} \delta\left(t_{k}-t_{j}\right), \forall k, j \in \mathbb{R}, \\
\mathbb{E}[\widetilde{v}(k)] & =0, \quad \mathbb{E}\left[\widetilde{v}(k) \widetilde{v}^{T}(j)\right]=\mathcal{I} \delta\left(t_{k}-t_{j}\right), \forall k, j \in \mathbb{R}, \widetilde{v}(k)=\mathcal{V}_{k}^{\frac{-1}{2}} v(k), \\
\mathbb{E}\left[v_{k}\right] & =0, \mathbb{E}\left[v_{k} v_{j}^{T}\right]=\mathcal{V}_{k} \delta\left(t_{k}-t_{j}\right), \forall k, j \in \mathbb{Z}_{+}, \\
\mathbb{E}\left[w_{2}(k) \widetilde{v}^{T}(j)\right] & =\mathbb{E}\left[w_{\infty}(k) \widetilde{v}^{T}(j)\right]=\mathbb{E}\left[w_{2}(k) \mathrm{x}^{T}(j)\right]=\mathbb{E}\left[\widetilde{v}(k) \mathrm{x}^{T}(j)\right]=0,
\end{aligned}\right.
$$

where $\delta(0)=1$ and $\delta(k)=0 ; \forall k \neq 0$. The known positive semi-definite assumption is considered for the matrices, $\mathcal{W}_{k_{\infty}}$ and $\mathcal{W}_{k_{2}}$. In addition, $\mathcal{V}_{k}=\operatorname{diag}\left(\lambda_{1}(k), \cdots, \lambda_{q}(k)\right)$, and $\lambda_{i}(k)$ is the variance of the independent noise of the $i^{t h}$ sensor measurement. The vector of the scheduling parameters is

$$
\xi(k)=\left[\xi_{1}(k), \cdots, \xi_{q}(k), \xi_{q+1}(k), \cdots, \xi_{n_{\xi}}(k)\right]^{T},
$$

where $\xi_{1}(k), \cdots, \xi_{q}(k)$ are scheduling parameters simulate the noises co-variance alteration of the sensors measurements; and $\xi_{q+1}(k), \cdots, \xi_{n_{\xi}}(k)$ are scheduling parameters simulate the alterations of system parameters. Practically, control system attributes may be shifted by the physical parameter alterations and sensor noise co-variance alterations. Therefore, an algorithm to online predicts the alteration of noise co-variance has been built in $[28,18,20]$. The innovation sequences being consistent with their theoretical co-variance is the core idea; see Refs. [28, 18, 20,19]. The vector $\xi(k)=\left[\xi_{1}(k), \cdots, \xi_{n_{\xi}}(k)\right]^{T}$ is proposed to be in a known a space $\Lambda_{n_{\xi}}$, which is polytopic, where $\xi_{i}(k) \in \Lambda, \forall i=$ $1, \cdots, n_{\xi}$. The bounds of the independent parameters are $\underline{\xi}_{i} \leq \xi_{i}(k) \leq \bar{\xi}_{i}, \quad \forall i=1, \cdots, n_{\xi} \quad \forall k \in \mathbb{Z}_{+}$, and they are known, where the lower and the upper bounds are $\underline{\xi}_{i}$ and $\bar{\xi}_{i}$, respectively, associated with the $i^{t h}$ parameter. Consequently, the rate of alteration of the $i^{t h}$ parameter is $\Delta_{\xi_{i}}(k)=\xi_{i}(k+1)-\xi_{i}(k), \forall i=1, \cdots, n_{\xi}, \forall k \in \mathbb{Z}_{+}$, with bounds $\underline{\Delta}_{\xi_{i}} \leq \Delta_{\xi_{i}}(k) \leq \bar{\Delta}_{\xi_{i}}, \quad \forall i=1, \cdots, n_{\xi}, \forall k \in \mathbb{Z}_{+}$, where the upper and lower bounds of the rate of alteration are $\bar{\Delta}_{\xi_{i}}$ and $\Delta_{\xi_{i}}$, respectively. The vector $\Delta_{\xi}(k)=\left[\Delta_{\xi_{1}}(k), \cdots, \Delta_{\xi_{n_{\xi}}}(k)\right]^{T} \in \Lambda_{n_{\xi}}$, where $\Delta_{\xi_{i}}(k) \in \Lambda, \forall i=1, \cdots, n_{\xi}$ denotes the parameters alteration rates, which yields $\sum_{i=1}^{n_{\xi}} \Delta_{\xi_{i}}=0$. The alteration rates bounds of parameter $\xi_{i}$ is $\left|\Delta_{\xi_{i}}\right| \leq a_{i} \in\{0,1\}, \forall i=1, \cdots, n_{\xi}$, In fact, the alteration rate depends on the associated parameter value for the discrete-time case. Considering the uncertainty space $\Pi_{a}$ contains the vector $\left[\xi^{T}(k), \Delta_{\xi}^{T}(k)\right]^{T} \in \Lambda_{2 n_{\xi}}$; see Ref.[19] for more details, each $\xi_{i}(k) \in \Lambda$ and $\Delta_{\xi_{i}}(k) \in \Lambda$, which are belong to $\xi(k)$, and $\Delta_{\xi}(k)$ vectors, respectively, are given as 
[19],

$$
\xi_{i}(k)=\sum_{j=1}^{M} f_{i}^{j} \widetilde{\xi}_{j}(k), \quad \Delta_{\xi_{i}}(k)=\sum_{j=1}^{M} h_{i}^{j} \widetilde{\xi}_{j}(k), \quad i=1, \cdots, n_{\xi},
$$

where $\widetilde{\xi}(k)=\left[\widetilde{\xi}_{1}(k), \cdots, \widetilde{\xi}_{M}(k)\right]^{T} \in \Pi_{a}$. Next, the PLMI-based synthesis conditions for designing multi-objective GS - DOF controllers are discussed.

\section{DYNAMIC OUTPUT FEEDBACK CONTROL SYNTHESIZE LMIS}

The DOF - LPV controller (which is strictly proper and full-order) is

$$
\mathcal{K}:=\left\{\begin{aligned}
\mathrm{x}_{c}(k+1) & =\mathcal{A}_{c}(\xi(k)) \mathrm{x}_{c}(k)+\mathcal{B}_{c}(\xi(k)) \mathrm{y}(k), \\
\mathrm{u}(k) & =\mathcal{C}_{c}(\xi(k)) \mathrm{x}_{c}(k),
\end{aligned}\right.
$$

the vector is $\mathrm{x}_{c}(k)$ designates the states of the controller; $\mathcal{A}_{c}(\xi(k)) \in \mathbb{R}^{n \times n} ; \mathcal{B}_{c}(\xi(k)) \in \mathbb{R}^{n \times q}$; and $\mathcal{C}_{c}(\xi(k)) \in \mathbb{R}^{m \times n}$ are controller parameter dependent matrices. Consider system (1), then the closed loop system,

$$
H_{c l}:=\left\{\begin{aligned}
\mathrm{x}_{c l}(k+1) & =\mathcal{A}_{c l}(\xi(k)) \mathrm{x}_{c l}(k)+\mathcal{B}_{2 c l}(\xi(k)) \widetilde{w}_{2 p}(k)+\mathcal{B}_{\infty c l}(\xi(k)) \widetilde{w}_{\infty p}(k), \\
\widetilde{\mathrm{z}}_{\infty}(k) & =\mathcal{C}_{\infty c l}(\xi(k)) \mathrm{x}_{c l}(k)+\mathcal{D}_{\infty c l}(\xi(k)) \widetilde{w}_{\infty p}(k) \\
\widetilde{\mathrm{z}}_{2}(k) & =\mathcal{C}_{2 c l}(\xi(k)) \mathrm{x}_{c l}(k)
\end{aligned}\right.
$$

where $\mathbf{x}_{c l}(k)=\left[\mathbf{x}(k)^{T} \mathbf{x}_{c}(k)^{T}\right]^{T} \in \mathbb{R}^{2 n}$ is the augmented state; and the matrices of the closed loop system are

$$
\begin{aligned}
& \mathcal{A}_{c l}(\xi(k))=\left[\begin{array}{cc}
\mathcal{A}(\xi(k)) & \mathcal{B}_{u}(\xi(k)) \mathcal{C}_{c}(\xi(k)) \\
\mathcal{B}_{c}(\xi(k)) \mathcal{C}_{y}(\xi(k)) & \mathcal{A}_{c}(\xi(k))
\end{array}\right], \quad \mathcal{B}_{2 c l}(\xi(k))=\left[\begin{array}{c}
\mathcal{B}_{2 w}(\xi(k)) \\
\mathcal{B}_{c}(\xi(k)) \mathcal{D}_{y v}
\end{array}\right],
\end{aligned}
$$

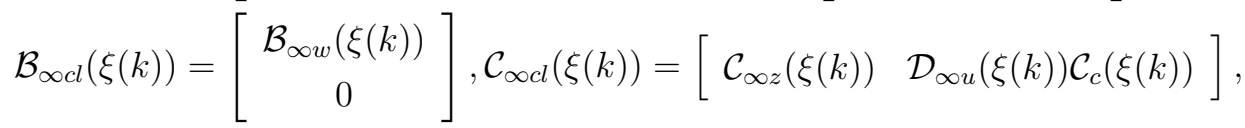

$$
\begin{aligned}
& \mathcal{D}_{\infty c l}(\xi(k))=\left[\begin{array}{cc}
\mathcal{D}_{\infty w}(\xi(k)) & 0
\end{array}\right], \mathcal{C}_{2 c l}(\xi(k))=\left[\begin{array}{ll}
\mathcal{C}_{2 z}(\xi(k)) & \mathcal{D}_{2 u}(\xi(k)) \mathcal{C}_{c}(\xi(k))
\end{array}\right], \\
& \widetilde{w}_{\infty p}(k)=\left[w_{\infty}^{T}(k) \widetilde{v}^{T}(k)\right]^{T}, \widetilde{w}_{2 p}(k)=\left[w_{2}^{T}(k) \widetilde{v}^{T}(k)\right]^{T} .
\end{aligned}
$$

The exponential stability inherited by system (7) regarding all permissible trajectories of $\xi(k)=\left[\xi_{1}(k), \cdots, \xi_{n_{\xi}}(k)\right]^{T} \in$ $\Lambda_{n_{\xi}}$. In the same manner, the upper bound on the $\mathrm{H}_{\infty} \& \mathrm{H}_{2}$ performances of the closed loop are guaranteed. To make the considered issue manageable, the $\mathrm{H}_{2}$-performance upper bound is optimized alternatively, exposed to the constraint on the $\mathrm{H}_{\infty}$-performance. The $\mathrm{H}_{2} \& \mathrm{H}_{\infty}$ performances are defined, such as, Considering that the system (7) has exponential stability, and the transfer matrix from $\widetilde{w}_{\infty p}(k) \in \ell_{2}^{r_{\infty w}+r_{\widetilde{v}}}$ to $\widetilde{\mathbf{z}}_{\infty}(k) \in \ell_{2}^{p_{\widetilde{ }}}$ is $\Re_{\widetilde{\mathbf{z}}_{\infty}, \widetilde{w}_{\infty p}}$. The $\mathrm{H}_{\infty}$-performance is [19]

$$
\left\|\Re_{\widetilde{\mathrm{z}}_{\infty}, \widetilde{w}_{\infty p}}(\xi(k))\right\|_{\infty}=\sup _{\left\|\widetilde{w}_{\infty p}(k)\right\|_{2} \neq 0} \frac{\left\|\widetilde{\mathbf{z}}_{\infty}(k)\right\|_{2}}{\mid \widetilde{w}_{\infty p}(k) \|_{2}}
$$

Then, the $\mathrm{H}_{\infty}$-performance (9) is upper bounded by

$$
\sup _{\xi(k) \in \Lambda_{n_{\xi}}}\left\|\mathcal{G}_{\widetilde{\mathrm{z}}_{\infty}, \widetilde{w}_{\infty p}}(\xi(k))\right\|_{\infty} \leq \alpha,
$$

where $\alpha$ is a positive scalar number. Considering that system (7) has exponential stability. The $\mathrm{H}_{2}$-performance is [19]

$$
\left\|\Re_{\widetilde{\mathrm{z}}_{2}, \widetilde{w}_{2 p}}(\xi(k))\right\|_{2}^{2}=\lim _{\mathcal{T} \rightarrow \infty} \sup _{\xi(k) \in \Lambda_{n_{\xi}}} \mathbb{E}\left\{\frac{1}{\mathcal{T}} \sum_{k=0}^{\mathcal{T}} \widetilde{\mathrm{z}}_{2}^{T}(k) \widetilde{\mathbf{z}}_{2}(k)\right\},
$$

where the transfer function matrix from $\widetilde{w}_{2 p}$ to $\widetilde{\mathrm{z}}_{2}$ is $\Re_{\widetilde{\mathrm{z}}_{2}, \widetilde{w}_{2 p}}$, the polytopic space, where the scheduling parameters be is $\Lambda_{n_{\xi}}$; the expectation operator is $\mathbb{E}$; and the positive integer $\mathcal{T}$ refers the time horizon. The formulation of the minimization of the upper bound of $\mathrm{H}_{2}$-performance exposed to a constraint on the $\mathrm{H}_{\infty}$-performance is provided by the PLMls set in the next a few subsections, where the PLMIs set has been invested in synthesizing a GS - DOF control for a polytopic DT - LPV system. 


\section{1. $\mathrm{H}_{\infty}$ Gain Scheduling DOF}

The following lemma states the $\mathrm{H}_{\infty}$-performance of system (7).

Lemma 1 Consider the GS - DOF control (6) and system (7). The later has $\mathrm{H}_{\infty}$-performance bound ( $\alpha$ ) if a matrix $\mathcal{P}_{\infty}(\xi) \in \mathbb{R}^{2 n \times 2 n}$ be in existence and a matrix $\mathcal{G}(\xi) \in \mathbb{R}^{2 n \times 2 n}$, which are symmetric positive-definite, such that, $\forall k \geq 0$, the following retains,

$$
\left[\begin{array}{cccc}
\mathcal{P}_{\infty}(\xi(k+1)) & \mathcal{A}_{c l}(\xi(k)) \mathcal{G}(\xi(k)) & \mathcal{B}_{\infty c l}(\xi(k)) & \mathbf{0} \\
\star & \mathcal{G}(\xi(k))+\mathcal{G}(\xi(k))^{T}-\mathcal{P}_{\infty}(\xi(k)) & \mathbf{0} & \mathcal{G}(\xi(k))^{T} \mathcal{C}_{\infty c l}(\xi(k))^{T} \\
\star & \star & \alpha I & \mathcal{D}_{\infty c l}(\xi(k))^{T} \\
\star & \star & \star & \alpha I
\end{array}\right]>0
$$

Remark 1 Clearly, substituting the matrices of system (8) into the inequality (12) causes a nonlinear inequality. To remedy this, a nonlinear mutation of variables is used. Furthermore, due to the fact that the slack variable $\mathcal{G}(\xi(k))$ appears in the nonlinear mutation of variables, parameter independent structure (i.e., $\mathcal{G}(\xi(k))=\mathcal{G})$ is mandatory.

\subsubsection{Nonlinear mutation of variables} are defined as

To deal with coupling between controller gain and plant matrices, the matrices $K(\xi(k)), \mathcal{P}(\xi(k)), \mathcal{G}$, and $\mathcal{G}^{-1}$

$$
\begin{aligned}
K(\xi(k)) & =\left[\begin{array}{cc}
\mathcal{A}_{c}(\xi(k)) & \mathcal{B}_{c}(\xi(k)) \\
\mathcal{C}_{c}(\xi(k)) & 0
\end{array}\right], \quad \mathcal{P}_{\infty}(\xi(k))=\left[\begin{array}{cc}
P_{\infty}(\xi(k)) & P_{2 \infty}(\xi(k)) \\
P_{2 \infty}(\xi(k))^{T} & P_{3 \infty}(\xi(k))
\end{array}\right], \\
\mathcal{G} & =\left[\begin{array}{ll}
\mathscr{X} & \mathscr{Z}_{1} \\
\mathscr{U} & \mathscr{Z}_{2}
\end{array}\right], \quad \mathcal{G}^{-1}=\left[\begin{array}{ll}
\mathscr{Y}^{T} & \mathscr{Z}_{3} \\
\mathscr{V}^{T} & \mathscr{Z}_{4}
\end{array}\right] .
\end{aligned}
$$

Considering the structure of $\mathcal{G}$ and $\mathcal{G}^{-1}$, obviously the next inequality must satisfy

$$
\mathcal{G G}^{-1}=\left[\begin{array}{cc}
\mathscr{X} & \mathscr{Z}_{1} \\
\mathscr{U} & \mathscr{Z}_{2}
\end{array}\right]\left[\begin{array}{ll}
\mathscr{Y}^{T} & \mathscr{Z}_{3} \\
\mathscr{V}^{T} & \mathscr{Z}_{4}
\end{array}\right]=\left[\begin{array}{ll}
\mathscr{X} \mathscr{Y}^{T}+\mathscr{Z}_{1} \mathscr{V}^{T} & \mathscr{X}_{3}+\mathscr{Z}_{1} \mathscr{Z}_{4} \\
\mathscr{U} \mathscr{Y}^{T}+\mathscr{Z}_{2} \mathscr{V}^{T} & \mathscr{U} \mathscr{Z}_{3}+\mathscr{Z}_{2} \mathscr{Z}_{4}
\end{array}\right]=\left[\begin{array}{ll}
I & 0 \\
0 & I
\end{array}\right],
$$

in a way that $\mathscr{X} \mathscr{Y}^{T}+\mathscr{Z}_{1} \mathscr{V}^{T}=I$ and $\mathscr{U} \mathscr{Y}^{T}+\mathscr{Z}_{2} \mathscr{V}^{T}=0$. The transformation matrix (which is parameter independent) is

$$
\mathbf{T}=\left[\begin{array}{ll}
I & \mathscr{Y}^{T} \\
0 & \mathscr{V}^{T}
\end{array}\right]
$$

Consequently, the parameter dependent nonlinear mutation of variables is introduced:

$$
\begin{aligned}
& {\left[\begin{array}{cc}
Q(\xi(k)) & F(\xi(k)) \\
L(\xi(k)) & 0
\end{array}\right]:=\left[\begin{array}{cc}
\mathscr{V} & \mathscr{Y} B_{u}(\xi(k)) \\
0 & I
\end{array}\right] K(\xi(k))\left[\begin{array}{cc}
\mathscr{U} & 0 \\
\mathcal{C}_{y}(\xi(k)) X & I
\end{array}\right]+\left[\begin{array}{c}
\mathscr{Y} \\
0
\end{array}\right] \mathcal{A}(\xi(k))\left[\begin{array}{ll}
\mathscr{X} & 0
\end{array}\right],} \\
& {\left[\begin{array}{cc}
P_{\infty}(\xi(k)) & J(\xi(k)) \\
J(\xi(k))^{T} & H(\xi(k))
\end{array}\right]:=\mathbf{T}^{T} \mathcal{P}_{\infty}(\xi(k)) \mathbf{T},} \\
& \quad S:=\mathscr{Y} \mathscr{X}+\mathscr{V} \mathscr{U} .
\end{aligned}
$$

Linearizing the transformation depends on identities:

$$
\begin{aligned}
\mathbf{T}^{T} \mathcal{A}_{c l} \mathcal{G} \mathbf{T} & =\left[\begin{array}{cc}
\mathcal{A}(\xi(k)) X+\mathcal{B}_{u}(\xi(k)) L(\xi(k)) & \mathcal{A}(\xi(k)) \\
Q(\xi(k)) & \mathscr{Y} \mathcal{A}(\xi(k))+F(\xi(k)) \mathcal{C}_{y}(\xi(k))
\end{array}\right], \\
\mathbf{T}^{T} \mathcal{B}_{\infty c l}(\xi(k)) & =\left[\begin{array}{c}
\mathcal{B}_{\infty w}(\xi(k)) \\
\mathscr{Y} \mathcal{B}_{\infty w}(\xi(k))+F(\xi(k)) \mathcal{D}_{y v}(\xi(k))
\end{array}\right], \\
\mathcal{C}_{\infty c l}(\xi(k)) \mathcal{G} \mathbf{T} & =\left[\begin{array}{cc}
\mathcal{C}_{\infty z}(\xi(k)) X+\mathcal{D}_{\infty u}(\xi(k)) L(\xi(k)) & \mathcal{C}_{\infty z}(\xi(k))
\end{array}\right] \\
\mathcal{D}_{\infty c l}(\xi(k)) & =\left[\begin{array}{cc}
\mathcal{D}_{\infty w}(\xi(k)) & 0
\end{array}\right] \\
\mathbf{T}^{T} \mathcal{G} \mathbf{T} & =\left[\begin{array}{cc}
\mathscr{X} & I \\
S & \mathscr{Y}
\end{array}\right] .
\end{aligned}
$$


Multiplying from the right of Eqn. (12) by $T:=\operatorname{diag}(\mathbf{T}, \mathbf{T}, I, I)$ and by $T^{T}$ from the left results the upcoming LMI with $P_{\infty}(\xi(k))$ and $H(\xi(k))$, which are symmetric positive-definite matrices, and matrices $\mathscr{X}, L(\xi(k)), \mathscr{Y}, F(\xi(k))$, $Q(\xi(k)), S, J(\xi(k))$ :

$$
\begin{gathered}
{\left[\begin{array}{cccccc}
P_{\infty}(\xi(k+1)) & J(\xi(k+1)) & \xi_{13}(\xi(k)) & \mathcal{A}(\xi(k)) & \mathcal{B}_{\infty w}(\xi(k)) & 0 \\
\star & H(\xi(k+1)) & Q(\xi(k)) & \xi_{24}(\xi(k)) & \xi_{25}(\xi(k)) & 0 \\
\star & \star & \mathscr{X}+\mathscr{X}^{T}-P_{\infty}(\xi(k)) & I+S^{T}-J(\xi(k)) & 0 & \xi_{36}(\xi(k)) \\
\star & \star & \star & \mathscr{Y}+\mathscr{Y}^{T}-H(\xi(k)) & 0 & \mathcal{C}_{\infty z}(\xi(k))^{T} \\
\star & \star & \star & \star & \alpha I & \mathcal{D}_{\infty w}(\xi(k))^{T} \\
\star & \star & \star & \star & \star & \alpha I
\end{array}\right]} \\
=\xi(\xi(k))>0,
\end{gathered}
$$

where,

$$
\begin{aligned}
& \xi_{13}(\xi(k))=\mathcal{A}(\xi(k)) \mathscr{X}+\mathcal{B}_{u}(\xi(k)) L(\xi(k)), \\
& \xi_{24}(\xi(k))=\mathscr{Y} \mathcal{A}(\xi(k))+F(\xi(k)) C_{y}(\xi(k)), \\
& \xi_{25}(\xi(k))=\mathscr{Y} \mathcal{B}_{\infty w}(\xi(k))+F(\xi(k)) \mathcal{D}_{y v}(\xi(k)), \\
& \xi_{36}(\xi(k))=\mathscr{X}^{T} \mathcal{C}_{\infty z}(\xi(k))^{T}+L(\xi(k))^{T} \mathcal{D}_{\infty u}(\xi(k))^{T} .
\end{aligned}
$$

If full ranking fit matrix $\mathscr{V}, \mathbf{T}$ will be the same, which leads to having a full rank $T$. If the PLMI in Eqn. (18) is held, a recovery of the gain scheduling $\mathrm{H}_{\infty}$ control done by inverting the transformation in Eqn. (16),

$$
K(\xi)=\left[\begin{array}{cc}
\mathscr{V}^{-1} & -\mathscr{V}^{-1} Y \mathcal{B}_{u}(\xi) \\
0 & I
\end{array}\right]\left[\begin{array}{cc}
Q(\xi)-\mathscr{Y} \mathcal{A}(\xi) X & F(\xi) \\
L(\xi) & 0
\end{array}\right]\left[\begin{array}{cc}
\mathscr{U}^{-1} & 0 \\
-\mathcal{C}_{y}(\xi) \mathscr{X}^{-1} & I
\end{array}\right]
$$

As a result, the controller matrices are

$$
\begin{aligned}
\mathcal{A}_{c}(\xi) & =\mathscr{V}^{-1}\left(Q(\xi)-\mathscr{Y} \mathcal{A}(\xi) \mathscr{X}-\mathscr{Y} B_{u}(\xi) L(\xi)-F(\xi) \mathcal{C}_{y}(\xi) X\right) \mathscr{U}^{-1} \\
\mathcal{B}_{c}(\xi) & =\mathscr{V}^{-1} F(\xi) \\
\mathcal{C}_{c}(\xi) & =L(\xi) \mathscr{U}^{-1}
\end{aligned}
$$

The following parameter dependent framework to the Lyapunov matrix $P(\xi(k))$ (i.e., both $P_{\infty}(\xi(k))$ and $\left.P_{2}(\xi(k))\right)$ and system parameter dependent matrices in Eqn. (8) is imposed to satisfy the requirements of a finite set of LMI conditions,

$$
P(\xi(k))=\sum_{i=1}^{n_{\xi}} \xi_{i}(k) P_{i}, \quad \xi(k) \in \Lambda_{n_{\xi}}
$$

Each $i^{t h}$ component of the scheduling parameters and their variation rate vectors, (i.e., $\xi_{i}(k)$ and $\Delta_{\xi_{i}}(k)$, respectively) can be given by

$$
\xi_{i}(k)=\sum_{j=1}^{M} f_{i}^{j} \widetilde{\xi}_{j}(k) \text { and } \Delta_{\xi_{i}}(k)=\sum_{i=1}^{M} h_{i}^{j} \widetilde{\xi}_{j}(k)
$$

such as

$$
\tilde{P}(\widetilde{\xi}(k))=\sum_{j=1}^{M} \widetilde{\xi}_{j}(k) \tilde{P}_{j}
$$

where the matrix $\tilde{P}_{j}=\sum_{i=1}^{n_{\xi}} f_{i}^{j} P_{i}$. Combining that with Eqn. (21) and $\xi(k+1)=\Delta_{\xi}(k)+\xi(k)$, yields

$$
\hat{P}(\widetilde{\xi}(k))=\sum_{j=1}^{M} \tilde{\xi}_{j}(k) \hat{P}_{j}
$$


where $\hat{P}_{j}=\sum_{i=1}^{n_{\xi}}\left(f_{i}^{j}+h_{i}^{j}\right) P_{i}$. Then, the LMI in Eqn. (18) is

$$
\left[\begin{array}{cccccc}
\hat{P}_{\infty}(\cdot) & \hat{J}(\cdot) & \tilde{\mathcal{A}}(\cdot) X+\tilde{\mathcal{B}}_{u}(\cdot) \tilde{L}(\cdot) & \tilde{\mathcal{A}}(\cdot) & \tilde{\mathcal{B}}_{\infty w}(\cdot) & 0 \\
\star & \hat{H}(\cdot) & \tilde{Q}(\cdot) & \mathscr{Y} \tilde{\mathcal{A}}(\cdot)+\tilde{F}(\cdot) \tilde{\mathcal{C}}_{y}(\cdot) & \mathscr{Y} \tilde{B}_{w}(\cdot)+\tilde{F}(\cdot) \tilde{\mathcal{D}}_{y v}(\cdot) & 0 \\
\star & \star & \mathscr{X}+\mathscr{X}^{T}-\tilde{P}_{\infty}(\cdot) & I+S^{T}-\tilde{J}(\cdot) & 0 & \mathscr{X}^{T} \tilde{\mathcal{C}}_{\infty z}(\cdot)^{T}+\tilde{L}(\widetilde{\xi})^{T} \tilde{\mathcal{D}}_{\infty u}(\cdot)^{T} \\
\star & \star & \star & \mathscr{Y}+\mathscr{Y}^{T}-\tilde{H}(\cdot) & 0 & \tilde{\mathcal{C}}_{\infty z}(\cdot)^{T} \\
\star & \star & \star & \star & \alpha I & \tilde{\mathcal{D}}_{\infty w}(\cdot)^{T} \\
\star & \star & \star & \star & \star & \alpha I
\end{array}\right]>0
$$

where, for simplicity, $(\cdot)=(\widetilde{\xi}(k))$ is used. Accordingly, the LMIs with finite-dimensional is expressed in next Lemma to synthesize the $\mathrm{H}_{\infty} / \mathrm{GS}-$ DOF control.

Lemma 2 Consider system (7) with a prescribed $\mathrm{H}_{\infty}$-performance upper bound ( $\alpha$ ), and given $f^{j}$ and $h^{j}$ vectors. If there exist $P_{i, \infty}, H_{i}$, which are symmetric positive-definite matrices, and $J_{i}, L_{i}, F_{i}, Q_{i}, S, \mathscr{Y}, \mathscr{X}$ matrices, such the following hold,

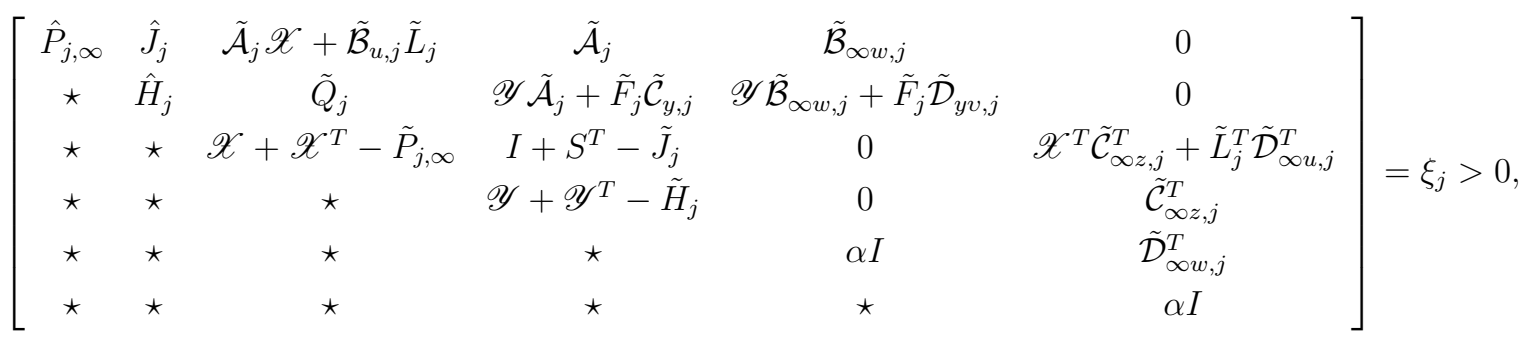

for $j=1, \ldots, M$

$$
\left[\begin{array}{cccccc}
\hat{P}_{j, \infty}+\hat{P}_{\ell, \infty} & \hat{J}_{j}+\hat{J}_{\ell} & \xi_{13, j \ell} & \tilde{\mathcal{A}}_{j}+\tilde{\mathcal{A}}_{\ell} & \tilde{\mathcal{B}}_{\infty w, j}+\tilde{\mathcal{B}}_{\infty w, \ell} & 0 \\
\star & \hat{H}_{j}+\hat{H}_{\ell} & \tilde{Q}_{j}+\tilde{Q}_{\ell} & \xi_{24, j \ell} & \xi_{25, j \ell} & 0 \\
\star & \star & 2 \mathscr{X}+2 \mathscr{X}^{T}-\tilde{P}_{j, \infty}-\tilde{P}_{\ell, \infty} & 2 I+2 S^{T}-\tilde{J}_{j}-\tilde{J}_{\ell} & 0 & \xi_{36, j \ell} \\
\star & \star & \star & 2 \mathscr{Y}+2 \mathscr{Y}^{T}-\tilde{H}_{j}-\tilde{H}_{\ell} & 0 & \tilde{\mathcal{C}}_{\infty z, j}^{T}+\tilde{\mathcal{C}}_{\infty z, \ell}^{T} \\
\star & \star & \star & \star & 2 \alpha I & \tilde{\mathcal{D}}_{\infty w, j}^{T}+\tilde{\mathcal{D}}_{\infty w, \ell}^{T} \\
\star & \star & \star & \star & \star & 2 \alpha I
\end{array}\right]
$$

$=\xi_{j \ell}>0$,

for $j=1, \ldots, M-1$ and $\ell=j+1, \ldots, M$ with

$$
\begin{aligned}
& \xi_{13, j \ell}=\tilde{\mathcal{A}}_{j} \mathscr{X}+\tilde{\mathcal{A}}_{\ell} \mathscr{X}+\tilde{\mathcal{B}}_{u, j} \tilde{L}_{\ell}+\tilde{\mathcal{B}}_{u, \ell} \tilde{L}_{j}, \\
& \xi_{24, j \ell}=\mathscr{Y} \tilde{\mathcal{A}}_{j}+\mathscr{Y} \tilde{\mathcal{A}}_{\ell}+\tilde{F}_{j} \tilde{\mathcal{C}}_{y, \ell}+\tilde{F}_{\ell} \tilde{\mathcal{C}}_{y, j}, \\
& \xi_{25, j \ell}=\mathscr{Y} \tilde{\mathcal{B}}_{\infty w, j}+\mathscr{Y} \tilde{\mathcal{B}}_{\infty w, \ell}+\tilde{F}_{j} \tilde{\mathcal{D}}_{y v, \ell}+\tilde{F}_{\ell} \tilde{\mathcal{D}}_{y v, j}, \\
& \xi_{36, j \ell}=\mathscr{X}^{T} \tilde{\mathcal{C}}_{\infty z, j}^{T}+\mathscr{X}^{T} \tilde{\mathcal{C}}_{\infty z, \ell}^{T}+\tilde{L}_{j}^{T} \tilde{\mathcal{D}}_{\infty u, \ell}^{T}+\tilde{L}_{\ell}^{T} \tilde{\mathcal{D}}_{\infty u, j}^{T},
\end{aligned}
$$


where

$$
\begin{aligned}
\hat{P}_{j, \infty} & =\sum_{i=1}^{n_{\xi}}\left(f_{i}^{j}+h_{i}^{j}\right) P_{i, \infty}, \quad \hat{J}_{j}=\sum_{i=1}^{n_{\xi}}\left(f_{i}^{j}+h_{i}^{j}\right) J_{i}, \quad \hat{H}_{j}=\sum_{i=1}^{n_{\xi}}\left(f_{i}^{j}+h_{i}^{j}\right) H_{i}, \\
\tilde{P}_{j, \infty}= & \sum_{i=1}^{n_{\xi}} f_{i}^{j} P_{i, \infty}, \quad \tilde{J}_{j}=\sum_{i=1}^{n_{\xi}} f_{i}^{j} J_{i}, \quad \tilde{H}_{j}=\sum_{i=1}^{n_{\xi}} f_{i}^{j} H_{i}, \\
\tilde{Q}_{j}= & \sum_{i=1}^{n_{\xi}} f_{i}^{j} Q_{i}, \quad \tilde{L}_{j}=\sum_{i=1}^{n_{\xi}} f_{i}^{j} L_{i}, \quad \tilde{F}_{j}=\sum_{i=1}^{n_{\xi}} f_{i}^{j} F_{i}, \quad \tilde{\mathcal{D}}_{y v, j}=\sum_{i=1}^{n_{\xi}} f_{i}^{j} \mathcal{D}_{y v, i} \\
\tilde{\mathcal{A}}_{j}= & \sum_{i=1}^{n_{\xi}} f_{i}^{j} \mathcal{A}_{i}, \quad \tilde{\mathcal{B}}_{\infty u, j}=\sum_{i=1}^{n_{\xi}} f_{i}^{j} \mathcal{B}_{\infty u, i}, \quad \tilde{\mathcal{B}}_{\infty w, j}=\sum_{i=1}^{n_{\xi}} f_{i}^{j} \mathcal{B}_{\infty w, i}, \quad \tilde{\mathcal{C}}_{y, j}=\sum_{i=1}^{n_{\xi}} f_{i}^{j} \mathcal{C}_{y, i}, \\
\tilde{\mathcal{C}}_{\infty z, j}= & \sum_{i=1}^{n_{\xi}} f_{i}^{j} \mathcal{C}_{\infty z, i}, \quad \tilde{\mathcal{D}}_{\infty u, j}=\sum_{i=1}^{n_{\xi}} f_{i}^{j} \mathcal{D}_{\infty u, i}, \quad \tilde{\mathcal{D}}_{\infty w, j}=\sum_{i=1}^{j} f_{i}^{j} \mathcal{D}_{\infty w, i} .
\end{aligned}
$$

Subsequently, the robustly parameter-dependent DOF control in Eqn. (6) stabilizes exponentially system (7) with ensured $\mathrm{H}_{\infty}$-performance upper bound $\alpha$.

The next subsection expresses the LMls set need to be satisfied to have $\mathbf{H}_{2} / G S-$ DOF control.

\section{2. $\mathrm{H}_{2}$ Gain Scheduling DOF}

The finite-dimensional LMIs set to have $\mathrm{H}_{2} / \mathrm{GS}$ - DOF control are only presented, to eliminate the redundancy by starting from PLMIs and then re-cast them in a finite-dimensional LMIs.

Lemma 3 Consider Eqn. (7) with a prescribed $f^{j}$ and $h^{j}$ vectors. If there exist matrices $P_{i, 2}, H_{i}$, which are symmetric positive-definite, and $\mathscr{W}_{i}, J_{i}, L_{i}, F_{i}, Q_{i}, S, Y, X$ matrices such that

$$
\left[\begin{array}{ccccc}
\hat{P}_{j, 2} & \hat{J}_{j} & \tilde{A}_{j} X+\tilde{B}_{u, j} \tilde{L}_{j} & \tilde{A}_{j} & \tilde{B}_{2 w, j} \\
\star & \hat{H}_{j} & \tilde{Q}_{j} & Y \tilde{A}_{j}+\tilde{F}_{j} \tilde{C}_{y, j} & Y \tilde{B}_{2 w, j}+\tilde{F}_{j} \tilde{D}_{y v, j} \\
\star & \star & X+X^{T}-\tilde{P}_{j, 2} & I+S^{T}-\tilde{J}_{j} & 0 \\
\star & \star & \star & Y+Y^{T}-\tilde{H}_{j} & 0 \\
\star & \star & \star & \star & I
\end{array}\right]=\Psi_{j}>0
$$

for $j=1, \ldots, M$

$$
\left[\begin{array}{ccccc}
\hat{P}_{j, 2}+\hat{P}_{\ell, 2} & \hat{J}_{j}+\hat{J}_{\ell} & \Psi_{13, j \ell} & \tilde{A}_{j}+\tilde{A}_{\ell} & \tilde{B}_{2 w, j}+\tilde{B}_{2 w, \ell} \\
\star & \hat{H}_{j}+\hat{H}_{\ell} & \tilde{Q}_{j}+\tilde{Q}_{\ell} & \Psi_{24, j \ell} & \Psi_{25, j \ell} \\
\star & \star & 2 X+2 X^{T}-\tilde{P}_{j, 2}-\tilde{P}_{\ell, 2} & 2 I+2 S^{T}-\tilde{J}_{j}-\tilde{J}_{\ell} & 0 \\
\star & \star & \star & 2 Y+2 Y^{T}-\tilde{H}_{j}-\tilde{H}_{\ell} & 0 \\
\star & \star & \star & \star & 2 I
\end{array}\right]=\Psi_{j \ell}>0
$$

for $j=1, \ldots, M-1$ and $\ell=j+1, \ldots, M$, with

$$
\begin{gathered}
\Psi_{13, j \ell}=\tilde{A}_{j} X+\tilde{A}_{\ell} X+\tilde{B}_{u, j} \tilde{L}_{\ell}+\tilde{B}_{u, \ell} \tilde{L}_{j} \\
\Psi_{24, j \ell}=Y \tilde{A}_{j}+Y \tilde{A}_{\ell}+\tilde{F}_{j} \tilde{C}_{y, \ell}+\tilde{F}_{\ell} \tilde{C}_{y, j} \\
\Psi_{25, j \ell}=Y \tilde{B}_{2 w, j}+Y \tilde{B}_{2 w, \ell}+\tilde{F}_{j} \tilde{D}_{y v, \ell}+\tilde{F}_{\ell} \tilde{D}_{y v, j}, \\
{\left[\begin{array}{ccc}
\tilde{\mathscr{W}}_{j}-\tilde{D}_{2 w, j} \tilde{D}_{2 w, j}^{T} & \tilde{C}_{2 z, j} X+\tilde{D}_{2 u, j} \tilde{L}_{j} & \tilde{C}_{2 z, j} \\
\star & X+X^{T}-\tilde{P}_{j, 2} & I+S^{T}-\tilde{J}_{j} \\
\star & \star & Y+Y^{T}-\tilde{H}_{j}
\end{array}\right]=\Phi_{j}>0,}
\end{gathered}
$$


for $j=1, \ldots, M$

$$
\begin{aligned}
& {\left[\begin{array}{ccc}
\tilde{\mathscr{W}}_{j}+\tilde{\mathscr{W}}_{\ell}-\tilde{D}_{2 w, j} \tilde{D}_{2 w, \ell}^{T}+\tilde{D}_{2 w, \ell} \tilde{D}_{2 w, j}^{T} & \tilde{C}_{2 z, j} X+\tilde{C}_{2 z, \ell} X+\tilde{D}_{2 u, j} \tilde{L}_{\ell}+\tilde{D}_{2 u, \ell} \tilde{L}_{j} & \tilde{C}_{2 z, j}+\tilde{C}_{2 z, \ell} \\
\star & 2 X+2 X^{T}-\tilde{P}_{j, 2}-\tilde{P}_{\ell, 2} & 2 I+2 S^{T}-\tilde{J}_{j}-\tilde{J}_{\ell} \\
\star & \star & 2 Y+2 Y^{T}-\tilde{H}_{j}-\tilde{H}_{\ell}
\end{array}\right]} \\
& =\Phi_{j \ell}>0,
\end{aligned}
$$

for $j=1, \ldots, M-1$ and $\ell=j+1, \ldots, M$, where,

$$
\begin{aligned}
\hat{P}_{j, 2} & =\sum_{i=1}^{n_{\xi}}\left(f_{i}^{j}+h_{i}^{j}\right) P_{i, 2}, \quad \hat{J}_{j}=\sum_{i=1}^{n_{\xi}}\left(f_{i}^{j}+h_{i}^{j}\right) J_{i}, \quad \hat{H}_{j}=\sum_{i=1}^{n_{\xi}}\left(f_{i}^{j}+h_{i}^{j}\right) H_{i}, \\
\tilde{P}_{j, 2} & =\sum_{i=1}^{n_{\xi}} f_{i}^{j} P_{i, 2}, \quad \tilde{J}_{j}=\sum_{i=1}^{n_{\xi}} f_{i}^{j} J_{i}, \quad \tilde{H}_{j}=\sum_{i=1}^{n_{\xi}} f_{i}^{j} H_{i}, \quad \tilde{\mathscr{W}}_{j}=\sum_{i=1}^{n_{\xi}} f_{i}^{j} \mathscr{W}_{i}, \\
\tilde{Q}_{j} & =\sum_{i=1}^{n_{\xi}} f_{i}^{j} Q_{i}, \quad \tilde{L}_{j}=\sum_{i=1}^{n_{\xi}} f_{i}^{j} L_{i}, \quad \tilde{F}_{j}=\sum_{i=1}^{n_{\xi}} f_{i}^{j} F_{i}, \quad \tilde{C}_{y, j}=\sum_{i=1}^{n_{\xi}} f_{i}^{j} C_{y, i}, \\
\tilde{A}_{j} & =\sum_{i=1}^{n_{\xi}} f_{i}^{j} A_{i}, \quad \tilde{B}_{2 u, j}=\sum_{i=1}^{n_{\xi}} f_{i}^{j} B_{2 u, i}, \quad \tilde{B}_{2 w, j}=\sum_{i=1}^{n_{\xi}} f_{i}^{j} B_{2 w, i}, \quad \tilde{D}_{y v, j}=\sum_{i=1}^{n_{\xi}} f_{i}^{j} D_{y v, i} \\
\tilde{C}_{2 z, j} & =\sum_{i=1}^{n_{\xi}} f_{i}^{j} C_{2 z, i}, \quad \tilde{D}_{2 u, j}=\sum_{i=1}^{n_{\xi}} f_{i}^{j} D_{2 u, i}, \quad \tilde{D}_{2 w, j}=\sum_{i=1}^{n_{\xi}} f_{i}^{j} D_{2 w, i} .
\end{aligned}
$$

Subsequently, the robustly parameter-dependent DOF control in Eqn. (6) stabilizes exponentially system (7) with ensured $\mathrm{H}_{2}$-performance upper bound $(\Gamma)$, as

$$
\Gamma^{2}=\min _{P_{i, 2}, H_{i}, \mathscr{W}_{i}, J_{i}, L_{i}, F_{i}, Q_{i}, S, Y, X} \max _{i} \operatorname{trace}\left(\mathscr{W}_{i}\right),
$$

\section{RESULT AND DISCUSSION}

The benefits of considering sensor senescence information during control design are expressed by simulations and numerical models, where constraints are imposed on the system outputs' performances. Two-fold of controllers are synthesized. A collection of the estimated noise co-variance and time-varying parameters is invested in scheduling a controller called fully gain scheduling. The other is scheduled by the latter only, which is called a partially gain scheduling. As a measure, the proportional improvement is considered to compare the fully gain scheduling control and partially one performances, such as

$$
\text { proportional improvement }=\frac{\mathrm{H}_{2}^{P G S}-\mathrm{H}_{2}^{F G S}}{\mathrm{H}_{2}^{P G S}} \times 100 \%,
$$

where the upper bounds of the $\mathrm{H}_{2}$-performance for the proposed fully and partially GS - DOF controls are $\mathrm{H}_{2}^{F G S}$ and $\mathrm{H}_{2}^{P G S}$, respectively. The computation complexity regarding the optimization process investing the linear matrix inequalities (LMls) set is [29]

$$
\text { computation complexity }=\log \left(\mathcal{J K}^{3}\right) \text {, }
$$

LPV Control Synthesis for Sensor Senescence

where the LMIs set have raws and scalar variables numbers denote by $\mathcal{J}$ and $\mathcal{K}$, respectively. Note that, MATLAB R2019 and semi-definite programming (SDP); solver SeDuMi [30]; interfaced by the parser YALMIP [31] have been invested to implement the simulation results. Example: consider system (1) with

$$
\left\{\begin{array}{c}
\mathcal{A}(\xi(k))=\left[\begin{array}{ccc}
-1+\xi_{1}(k)+\xi_{2}(k) & 0 & 1 \\
1 & 0.5 & 0 \\
0 & 1 & -0.5
\end{array}\right], \mathcal{B}_{u}(k)=\left[\begin{array}{l}
1 \\
0 \\
0
\end{array}\right], \mathcal{C}_{\infty z}(k)=\left[\begin{array}{lll}
1 & 0 & 0
\end{array}\right], \\
\mathcal{B}_{\infty w}=\mathcal{B}_{2 w}=\left[\begin{array}{l}
1 \\
0 \\
0
\end{array}\right], \mathcal{C}_{y}=\left[\begin{array}{lll}
1 & 0 & 0
\end{array}\right], \mathcal{D}_{\infty u}=\mathcal{D}_{2 u}=[1], \mathcal{C}_{2 z}=\left[\begin{array}{lll}
1 & 1 & 1
\end{array}\right],
\end{array}\right.
$$


Table 1. Positions of System (36) poles (i.e., open loop) along a broad extent of scheduling parameters.

\begin{tabular}{|c|c|c|c|c|}
\hline & $\xi_{1}=-0.2$ & $\xi_{1}=-0.2$ & $\xi_{1}=0.2$ & $\xi_{1}=0.2$ \\
\hline & $\xi_{2}=10^{-7}$ & $\xi_{2}=0.9$ & $\xi_{2}=10^{-7}$ & $\xi_{2}=0.9$ \\
\hline pole $_{1}$ & 0.8579 & 1.0074 & 0.9131 & 1.1125 \\
\hline pole $_{2}$ & $-1.0289+0.6758 i$ & $-0.6537+0.7999 i$ & $-0.8565+0.7619 i$ & $-0.5062+0.7875 i$ \\
\hline pole $_{3}$ & $-1.0289-0.6758 i$ & $-0.6537-0.7999 i$ & $-0.8565-0.7619 i$ & $-0.5062-0.7875 i$ \\
\hline
\end{tabular}

Table 2. Poles position of closed loop system along a broad extent of scheduling parameters, where the full GS - DOF controller is considered.

\begin{tabular}{|l|c|c|c|c|}
\hline & $\xi_{1} \in\{-0.2,0.2\}$, & $\xi_{1} \in\{-0.2,0.2\}$, & $\xi_{1} \in\{-0.2,0.2\}$, & $\xi_{1} \in\{-0.2,0.2\}$, \\
\hline & $\xi_{2} \in\left\{10^{-7}, 10^{-4}\right\}$ & $\xi_{2} \in\left\{10^{-7}, 10^{-2}\right\}$ & $\xi_{2} \in\left\{10^{-7}, 0.2\right\}$ & $\xi_{2} \in\left\{10^{-7}, 0.9\right\}$ \\
\hline pole $_{1}$ & 0.0663 & 0.0663 & $0.1364+0.0318 i$ & $0.3979+0.0506 i$ \\
\hline pole $_{2}$ & -0.0353 & -0.0358 & $0.1364-0.0318 i$ & $0.3979-0.0506 i$ \\
\hline pole $_{3}$ & 0.0081 & 0.0080 & $-0.0519+0.0986 i$ & $-0.2340+0.4302 i$ \\
\hline pole $_{4}$ & $0.0081-0.0042 i$ & $0.0080-0.0050 i$ & $-0.0519-0.0986 i$ & $-0.2340-0.4302 i$ \\
\hline pole $_{5}$ & -0.0050 & -0.0046 & $-0.0670+0.0165 i$ & $-0.2059+0.1054 i$ \\
\hline pole $_{6}$ & $-0.0050-0.0001 i$ & -0.0050 & $-0.0670-0.0165 i$ & $-0.2059-0.1054 i$ \\
\hline
\end{tabular}

where the bounds $\xi_{1}(k) \in[-0.2,0.2], \xi_{2}(k) \in\left[10^{-7}, 0.9\right]$ are imposed to the time-varying parameters. The scheduling parameters variations' rates, $\Delta \xi_{1}(\cdot)$ and $\Delta \xi_{2}(\cdot)$, are assigned to 0.02 and 0.002 , respectively. Correspondingly, $\xi_{1}(k)=$ $0.2 \sin (0.01 * k)$ and $\xi_{2}(k)=0.15 \cos (0.001 * k)+0.15$ are the scheduling parameters' pattern. The considered system above was firstly employed in [32] and later in [33], and it re-considered here as a state-of-art in standing literature, and to provide a fair comparison to these in existing LPV literature. Finally, a soft modification is made to the model to address the sensor senescence and demonstrate the proposed approach. Evoke that the GS - DOF control syntheses is the desired target, where the controller minimizes the upper bound of the system output $\mathrm{H}_{2}$-performance imposes to the constraint on the $\mathrm{H}_{\infty}$-performance bound. In addition, $\alpha=100$ is the assigned $\mathrm{H}_{\infty}$-performance upper bound. A line-search optimization technique is invested in obtaining this value, and it gives a feasible solution employing the SDP, SeDuMi along with YALMIP. Indeed, regarding the considered problem, the value near the minimum feasible value is $\alpha=100$. A worthy contrast between the GS - DOF controller and a conventional one is that the conventional controller dynamic matrices are parameter independent. Comparatively, the GS - DOF controller matrices in Eqn. (6) are parameter dependent matrices. Specifically, GS - DOF controller parameter dependent matrices are a convex collection for the parameter value at that moment, the DOF controller matrices computed at the vertices of the system for the imposed parameters and their alteration rates domains. Consequently, stack of matrices are computed for system performance in different application fields, as [9], transmission lines [10, 11], wireless sensor networks [12, 13], high-voltage systems [14]. For instance, utilizing Lemmas 3 and 2, system (36); and considering fully GS - DOF controller (6), where the sensor senescence is set to 0.9 , the robustly synthesized DOF controller matrices are

$$
\begin{aligned}
& \mathcal{A}_{c 1}= {\left[\begin{array}{ccc}
0.0321 & -1.6781 & -0.3603 \\
0.6125 & -0.7683 & -0.2779 \\
-0.1500 & 0.0759 & -0.3946
\end{array}\right] \quad \mathcal{A}_{c 2}=\left[\begin{array}{ccc}
0.0396 & -2.3966 & -0.5935 \\
0.6127 & -0.9282 & -0.3273 \\
-0.1497 & 0.0576 & -0.4024
\end{array}\right] } \\
& \mathcal{A}_{c 3}=\left[\begin{array}{ccc}
0.0428 & -2.6228 & -0.6669 \\
0.6119 & -0.9549 & -0.3358 \\
-0.1492 & 0.0441 & -0.4068
\end{array}\right] \quad \mathcal{A}_{c 4}=\left[\begin{array}{ccc}
0.0500 & -3.9998 & -1.1003 \\
0.6024 & -1.2441 & -0.4277 \\
-0.1465 & -0.0336 & -0.4304
\end{array}\right] \\
& \mathcal{B}_{c 1}=\left[\begin{array}{ll}
0.0141 \\
0.0079 \\
0.0045
\end{array}\right] \mathcal{B}_{c 2}=\left[\begin{array}{l}
0.0198 \\
0.0092 \\
0.0046
\end{array}\right] \quad \mathcal{B}_{c 3}=\left[\begin{array}{ll}
0.0216 \\
0.0094 \\
0.0047
\end{array}\right] \quad \mathcal{B}_{c 4}=\left[\begin{array}{ll}
0.0327 \\
0.0117 \\
0.0053
\end{array}\right] \\
& \mathcal{C}_{c 1}=\left[\begin{array}{lll}
-41.3546 & -51.1791 & -106.5410
\end{array}\right] \quad \mathcal{C}_{c 2}=\left[\begin{array}{lll}
-61.8772 & -49.1910 & -100.3390
\end{array}\right] \\
& \mathcal{C}_{c 3}=\left[\begin{array}{llll}
-68.5629 & -48.5762 & -98.1815
\end{array}\right] \mathcal{C}_{c 4}=\left[\begin{array}{lll}
-87.4414 & -45.4387 & -90.6900
\end{array}\right]
\end{aligned}
$$

For the system poles' locations, Table. 1 shows the positions of the open-loop system poles upon a broad extent of parameters. Obviously, the open-loop system owns unstable poles upon a vast extent of parameters. The full gain scheduling control is taken into account for the location of the closed loop poles. Table. 2 shows the positions of those poles for multiple values of the scheduling parameters, where they are located within a unit circle. Regarding the system performance, Fig. 1 shows the upper limit of the $\mathrm{H}_{2}$-performance like a function of the sensor senescence for full and partial GS - DOF controls. Fig. 2 shows the proportional improvement defined in Eqn. (34) for the proposed GS - DOF controllers. Those figures have motivating explications: as the sensor becomes senescence, the system performance to fulfill the imposed constraints gets worse. Besides, for the imposed sensor senescence information, the upper bound of the $\mathrm{H}_{2}$ - 


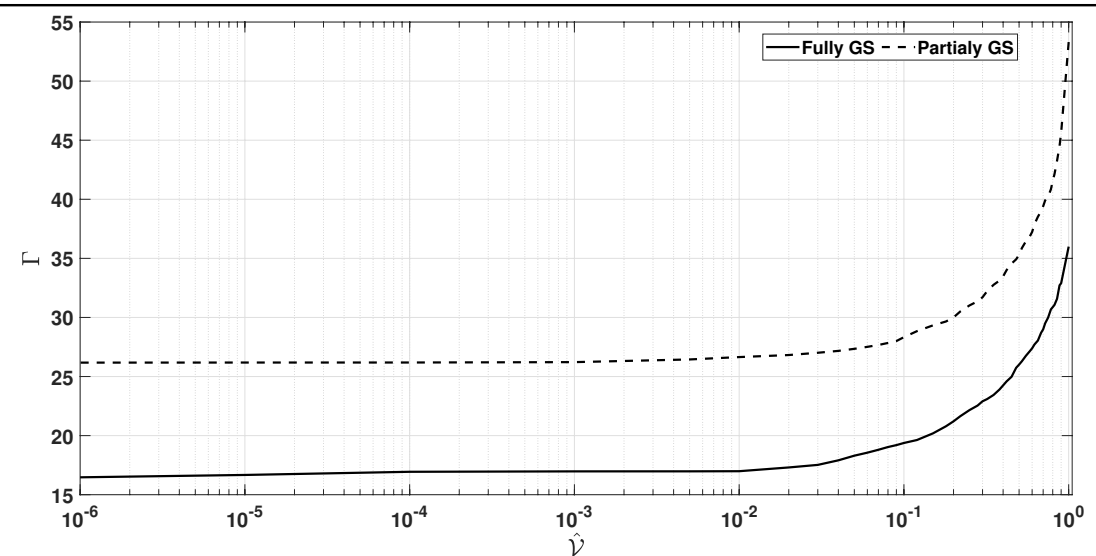

Fig. 1. The upper limit of the $\mathrm{H}_{2}$-performance (i.e., $\Gamma$ in Eqn.(33)) like a function of the sensor senescence in terms of the estimated co-variance of the sensor measurements noise (i.e., $\hat{\mathcal{V}}$ ) for full and partial GS - DOF controls.

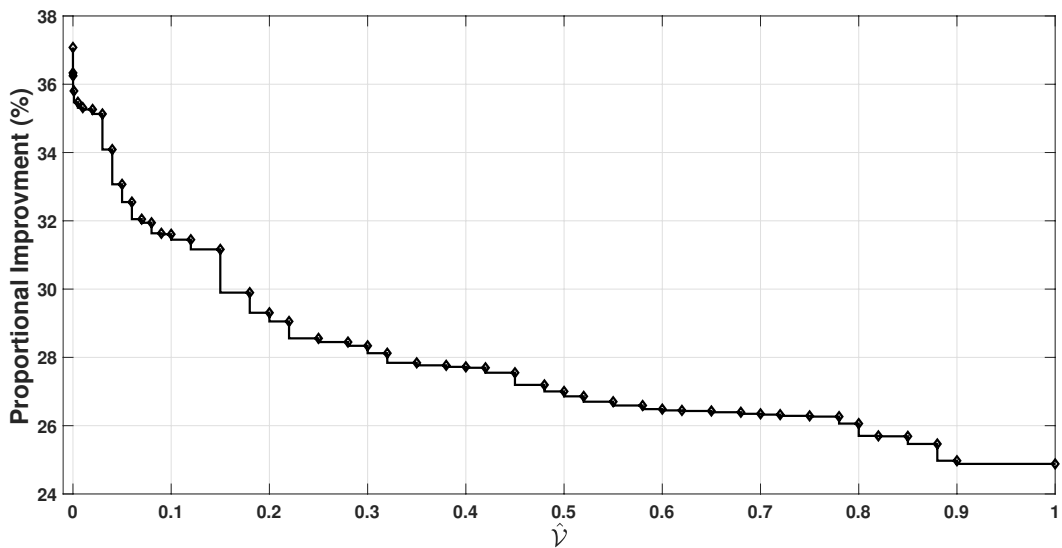

Fig. 2. The proportional improvement defined in Eqn. (34) like a function of the sensor senescence in terms of the estimated co-variance of the sensor measurements noise (i.e., $\hat{\mathcal{V}}$ ) for the synthesized GS - DOF controllers.

performance can be realized under the $\mathrm{H}_{\infty}$-performance upper bound. Moreover, the synthesized full GS - DOF control compensates the impact of slow-deterioration of sensor effectiveness due to senescence and guards the coveted performance. Otherwise, the partial GS controller attempts to compete with full GS for a while and then starts to retreat. To clarify, the designed full GS - DOF control has better performance over the partial one since the scheduling parameters only schedule the latter due to sensors senescence information. Finally, the closed loop system's performance with all GS - DOF controls deteriorates with fairly senescent sensors. Equally important, considering the full GS - DOF control and Lemmas 3 and 2, the designed controller effort demonstrates in terms of the $L_{2}$-norm of the Lyapunov matrix, $\mathrm{P}_{\mathrm{opt}}$, that satisfies Lemmas 3 and 2, while achieved control effort presents in terms of the $L_{2}$-norm of the solution of discrete-time Lyapunov matrix equation, $\mathrm{P}_{\text {lap }}$. Fig. 3 shows the designed and achieved controller efforts expressed by the $L_{2}$-norm of the Lyapunov matrix (i.e., $\left\|\mathrm{P}_{\text {opt }}\right\|_{2}$ and $\left\|\mathrm{P}_{\text {lap }}\right\|_{2}$, respectively) as a function of the sensor senescence. Clearly, the synthesized full GS - DOF control has achieved-effort less than the designed one for the considered system overall vertices, since the fully GS control scheduled by a combination of scheduling parameters due to system parameters and sensors senescence information. Touching with the computation complexity evaluation, Fig. 4 presents the computation complexity, and it shows that a full GS - DOF control has the highest computation complexity, while partially one has the lowest complexity. Regarding the system response to initial conditions. The time response for system (36) states have been shown in Fig. 5, considering full GS - DOF control, where sensor senescence information (i.e., noise co-variance) is imposed to 0.9. Worth mentioning that the poles number in the upper right sub-figures is six, which are calculated at the closed loop system vertices (i.e., vertices of the augmented system of the plant and controller); see Table 2 for details. Those figures have impressing interpretation: when the sensor senescence grows, the response becomes slower. That is, the designed GS - DOF control requires more period to compensate for the sensor senescence impact. Next, a summarization of the current work is presented.

\section{CONCLUSION}

In this work, a robust multi-objective GS - DOF control for LPV systems with sensor effectiveness degeneration caused by sensor senescence is addressed, where the synthesized gain scheduling (GS) dynamic output feedback (DOF) controller is imposed to a constraint on the $\mathrm{H}_{\infty}$-performance output. The recent literature considers the output measure- 

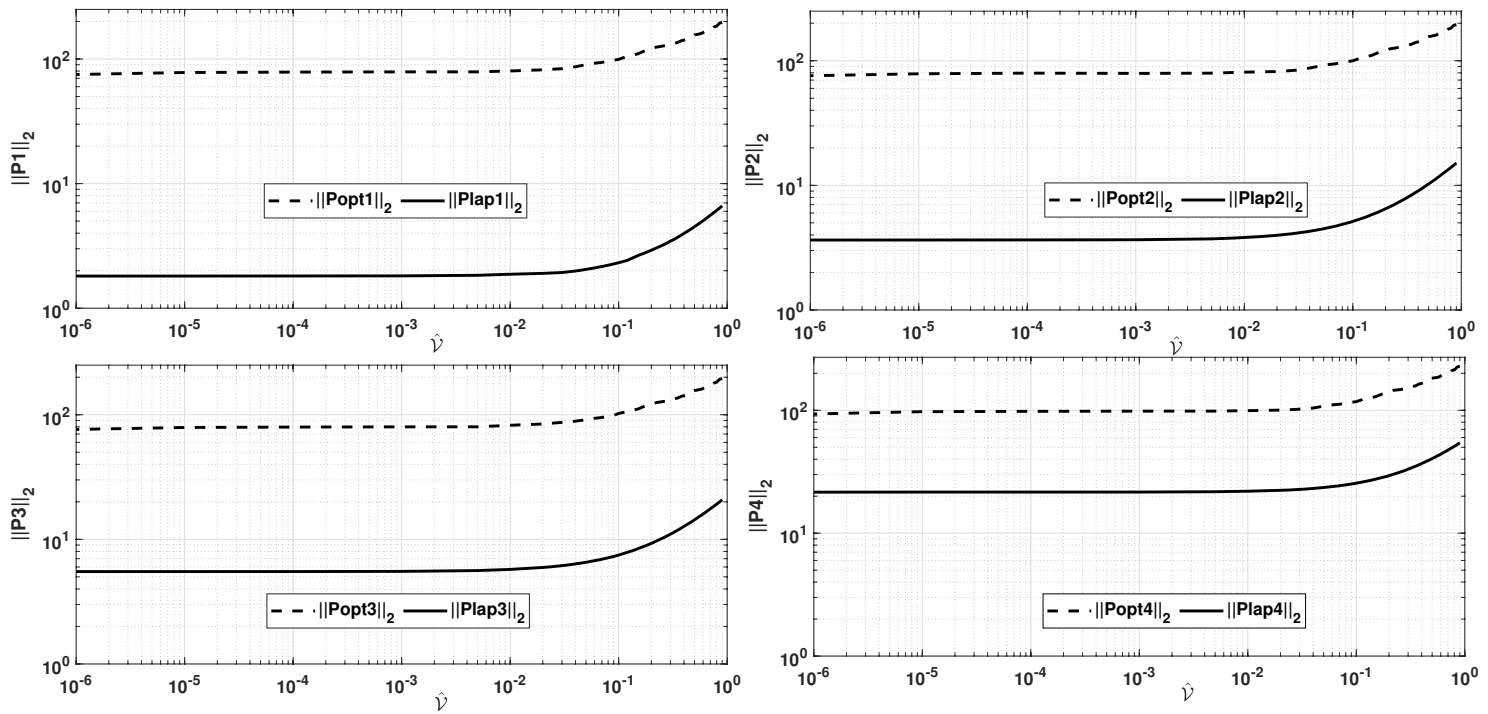

Fig. 3. The designed and achieved controller efforts expressed by the $L_{2}$-norm of the Lyapunov matrix (i.e., $\left\|\mathrm{P}_{\text {opt }}\right\|_{2}$ and $\left\|\mathrm{P}_{\text {lap }}\right\|_{2}$, respectively) as a function of the sensor senescence (i.e., co-variance of the sensor measurements noise $\hat{\mathcal{V}}$ ), where the solid and dotted lines associated with achieved and designed Lyapunov matrix $L_{2}$-norm, respectively.

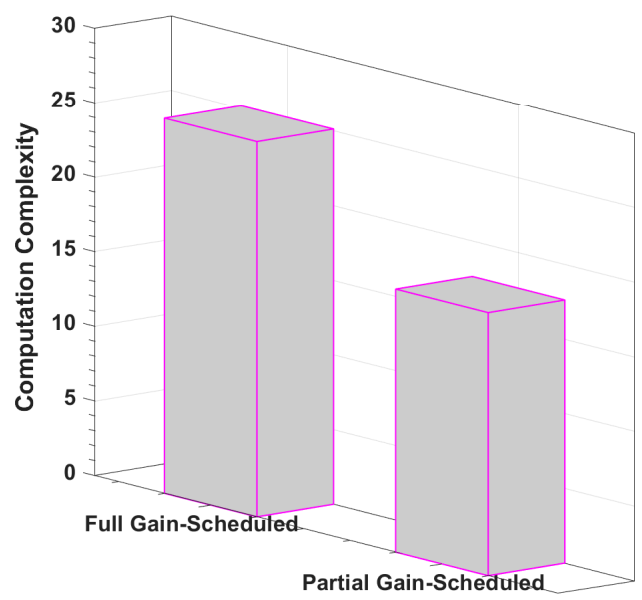

Fig. 4. Computation complexity (i.e., Eqn.(35)) comparison between synthesized GS - DOF controls.
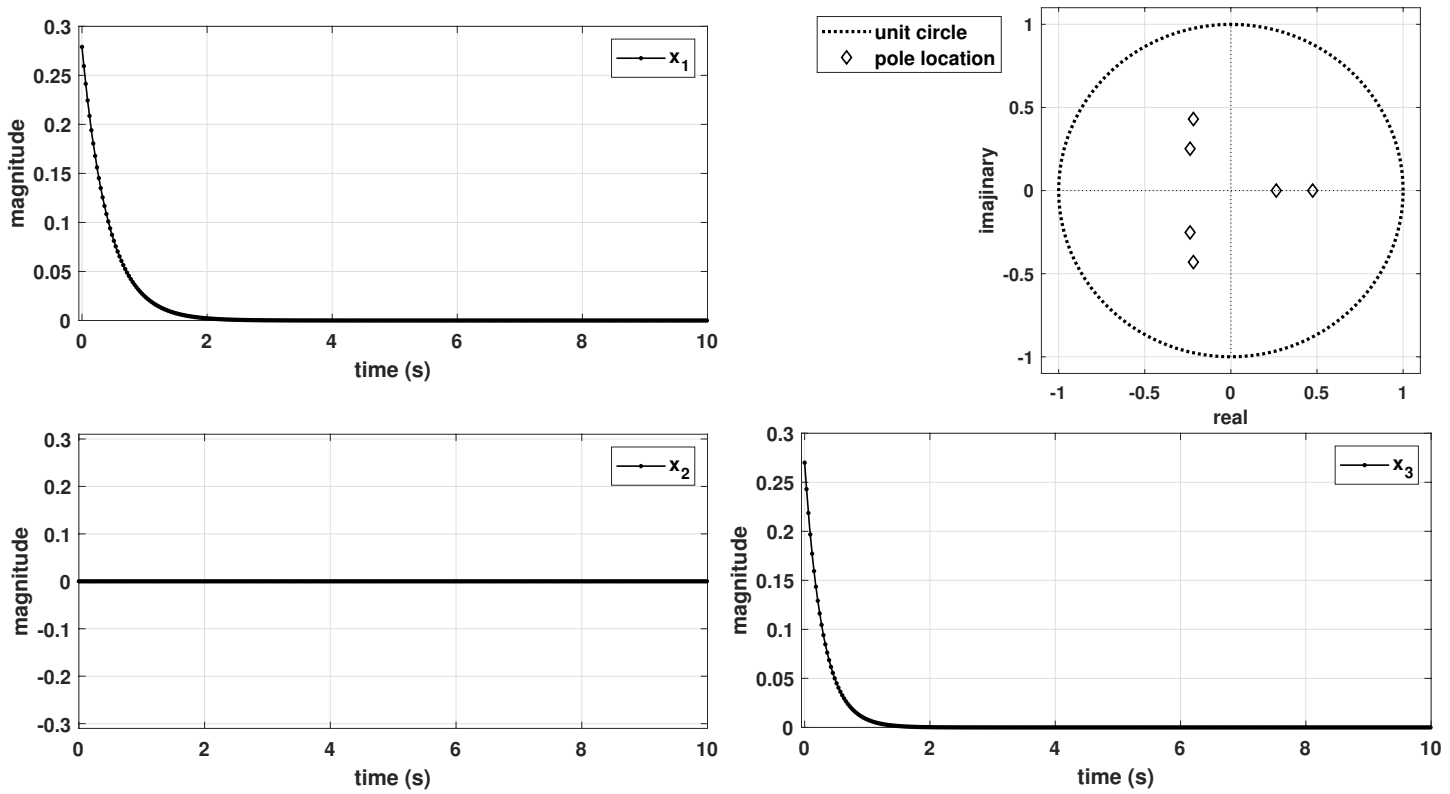

Fig. 5. Closed-loop system poles (upper right) and closed loop system initial condition response (rest of the sub-figures) for the considered system with fully gain scheduling control, where the noise co-variance is imposed to be 0.9 . 
ment may (or not) be affected by a known and time-invariant statistical characteristics noise, that is, assuming that sensor performance degradation as a fixed term is a conservative assumption. This paper considers that sensor effectiveness degeneration due to sensor senescence is a dynamical process, and it is characterized by the time-varying noise co-variance of the sensor measurements. Utilizing that, a multi-objective GS - DOF controller is designed using the LPV framework as well, as two sorts of controllers are synthesized. That is, a full gain scheduling (GS) controller is scheduled by a blend of the time-varying parameters, which are caused by the variation of system parameters and information of the sensor senescence alteration. A partial gain scheduling (GS) controller is scheduled only by the time-varying parameters caused by the sensor senescence information alteration. The layout of multi-objective GS - DOF control is formulated as an integrated $\mathrm{H}_{2} \& \mathrm{H}_{\infty}$ control issue that reduces the upper bound of system output $\mathrm{H}_{2}$-performance exposed to a constraint on the $\mathrm{H}_{\infty}$-performance. This issue can be remedied effectively by employing LMI optimization mechanisms. The designed DOF controller invests the sensor senescence information to minimize its impact upon the system and enhances the closed loop system performance. The synthesized multi-objective GS - DOF controller remedies for deteriorating sensor effectiveness caused by senescence and preserves the aimed performance. In addition, the synthesized control ensures the closed loop system stability. The simulations indicate that the synthesized full GS control does better than the partial one regarding the performance with steadily deteriorating sensor performance. Equally, the controller's performance gets worse when the sensor senescence increases significantly. Experimental validation is considered as an extension of future work of the current one.

\section{ACKNOWLEDGMENT}

The author greatly appreciates constructive comments from the editor and reviewers that are beneficial to improve the quality of the paper.

\section{REFERENCES}

[1] J. Mohammadpour and C. W. Scherer, Control of linear parameter varying systems with applications. Springer Science \& Business Media, New York, 2012.

[2] W. J. Rugh and J. S. Shamma, "Research on gain scheduling,” Automatica, vol. 36, no. 10, pp. 1401-1425, 2000.

[3] R. C. Oliveira, M. C. de Oliveira, and P. L. Peres, "LMI relaxations for robust $H_{2}$ performance analysis of polytopic linear systems," in Proceedings of the 45th IEEE Conference on Decision and Control. IEEE, 2006, pp. $2907-2912$.

[4] V. F. Montagner, R. C. Oliveira, and P. L. Peres, "Design of $H_{\infty}$ gain-scheduled controllers for linear time-varying systems by means of polynomial lyapunov functions," in Proceedings of the 45th IEEE Conference on Decision and Control. IEEE, 2006, pp. 5839-5844.

[5] J. M. Palma, C. F. Morais, and R. C. Oliveira, " $H_{2}$ control and filtering of discrete-time LPV systems exploring statistical information of the time-varying parameters," Journal of the Franklin Institute, vol. 357, no. 6, pp. 38353864, 2020.

[6] L. Frezzatto, M. J. Lacerda, R. C. Oliveira, and P. L. Peres, “ $H_{2}$ and $H_{\infty}$ fuzzy filters with memory for takagi-sugeno discrete-time systems,” Fuzzy Sets and Systems, vol. 371, pp. 78-95, 2019.

[7] A. L. Bertolin, P. L. Peres, and R. C. Oliveira, "Robust stability, $H_{2} / H_{\infty}$ guaranteed costs for discrete timevarying uncertain linear systems with constrained parameter variations," in 2019 American Control Conference (ACC). IEEE, 2019, pp. 4541-4546.

[8] J. M. P. Olate, L. de Paula Carvalho, T. E. Rosa, C. de Freitas Morais, and R. C. Oliveira, " $H_{2}$ and $H_{\infty}$ state-feedback control through multi-hop networks: Trade-off analysis between the network load and performance degradation," IEEE Latin America Transactions, vol. 16, no. 9, pp. 2377-2384, 2018.

[9] K. D. Kolavennu and A. G. Shaik, "Detection and discrimination of islanding and faults in distribution system with distributed generation by using wavelet based alienation approach," Indonesian Journal of Electrical Engineering and Informatics (IJEEI), vol. 7, no. 3, pp. 405-414, 2019.

[10] A. Azriyenni, M. Mustafa, D. Sukma, and M. Dame, "Backpropagation neural network modeling for fault location in transmission line 150 kv," Indonesian Journal of Electrical Engineering and Informatics (IJEEI), vol. 2, no. 1, pp. 1-12, 2014.

[11] N. Ghaffarzadeh, "A new method for recognition of arcing faults in transmission lines using wavelet transform and correlation coefficient," Indonesian Journal of Electrical Engineering and Informatics (IJEEI), vol. 1, no. 1, pp. 1-7, 2013. 
[12] S. Gupta and G. Gupta, "Simulation time and energy test for topology construction protocol in wireless sensor networks," Indonesian Journal of Electrical Engineering and Informatics (IJEEI), vol. 3, no. 2, pp. 89-92, 2015.

[13] S. Saxena, "Extension to hirloc algorithm for localization error computation in wireless sensor networks," Indonesian Journal of Electrical Engineering and Informatics (IJEEI), vol. 1, no. 4, pp. 119-126, 2013.

[14] S. Deb and H. Verma, "Effect of dc filters on selection of fault discriminator for unit protection of monopolar hvdc transmission system," Indonesian Journal of Electrical Engineering and Informatics (IJEEI), vol. 7, no. 3, pp. 393404, 2019.

[15] D. Rotondo, F. Nejjari, and V. Puig, "A virtual actuator and sensor approach for fault tolerant control of LPV systems," Journal of Process Control, vol. 24, no. 3, pp. 203-222, 2014.

[16] M. M. Seron and J. A. De Doná, "Robust fault estimation and compensation for LPV systems under actuator and sensor faults," Automatica, vol. 52, pp. 294-301, 2015.

[17] X. Liang, Q. Wang, C. Hu, and C. Dong, “Observer-based $H_{\infty}$ fault-tolerant attitude control for satellite with actuator and sensor faults," Aerospace Science and Technology, vol. 95, no. 1, p. 105424, 2019.

[18] A. Madhag and G. Zhu, "Online covariance estimation of slow-varying measurement noises using a modified adaptive filter," International Journal of Automation and Control, vol. 14, no. 2, pp. 187-212, 2020.

[19] A. Madhag, "Gain-scheduled control based on online estimated sensor aging," Ph.D. dissertation, Michigan State University, 2019.

[20] A. Madhag and G. Zhu, "Online sensor performance monitoring and fault detection for discrete linear parameter varying systems," International Journal of Automation and Control, vol. 14, no. 4, pp. 494-517, 2020. [Online]. Available: https://www.inderscience.com/info/ingeneral/forthcoming.php?jcode=IJAAC

[21] J. De Caigny, J. F. Camino, R. C. Oliveira, P. L. Peres, and J. Swevers, "Gain-scheduled dynamic output feedback control for discrete-time LPV systems," International Journal of Robust and Nonlinear Control, vol. 22, no. 5, pp. $535-558,2012$.

[22] J. De Caigny, J. F. Camino, R. C. Oliveira, P. L. D. Peres, and J. Swevers, "Gain-scheduled $H_{2}$ and $H_{\infty}$ control of discrete-time polytopic time-varying systems," IET control theory \& applications, vol. 4, no. 3, pp. 362-380, 2010.

[23] C. M. Agulhari, E. S. Tognetti, R. C. Oliveira, and P. L. Peres, " $H_{\infty}$ dynamic output feedback for LPV systems subject to inexactly measured scheduling parameters," in 2013 American Control Conference. IEEE, 2013, pp. 6060-6065.

[24] T. E. Rosa, C. F. Morais, and R. C. Oliveira, "New robust LMI synthesis conditions for mixed gain-scheduled reduced-order dof control of discrete-time lpv systems," International Journal of Robust and Nonlinear Control, vol. 28, no. 18, pp. 6122-6145, 2018.

[25] M. F. Braga, C. F. Morais, E. S. Tognetti, R. C. Oliveira, and P. L. Peres, "Discretization and discrete-time output feedback control of linear parameter varying continuous-time systems," in 53rd IEEE Conference on Decision and Control. IEEE, 2014, pp. 4765-4771.

[26] J. De Caigny, J. F. Camino, R. C. Oliveira, P. L. Peres, and J. Swevers, "Gain-scheduled dynamic output feedback control for discrete-time LPV systems," International Journal of Robust and Nonlinear Control, vol. 22, no. 5, pp. $535-558,2012$.

[27] V. Montagner, R. Oliveira, P. Peres, and P.-A. Bliman, "Linear matrix inequality characterisation for $H_{2}$ and $H_{\infty}$ guaranteed cost gain-scheduling quadratic stabilisation of linear time-varying polytopic systems," IET Control Theory \& Applications, vol. 1, no. 6, pp. 1726-1735, 2007.

[28] A. Madhag and G. G. Zhu, "Online sensor noise covariance identification using a modified adaptive filter," in ASME 2017 Dynamic Systems and Control Conference. American Society of Mechanical Engineers, 2017, pp. V002T04A001-V002T04A001.

[29] S. Boyd and L. Vandenberghe, Convex optimization. C Cambridge university press, New York, 2004.

[30] J. F. Sturm, "Using SeDuMi 1.02, a MATLAB toolbox for optimization over symmetric cones," Optimization methods and software, vol. 11, no. 1-4, pp. 625-653, 1999. 
[31] J. Lofberg, "Yalmip: A toolbox for modeling and optimization in matlab," in Computer Aided Control Systems Design, 2004 IEEE International Symposium on. IEEE, 2004, pp. 284-289.

[32] M. De Oliveira, J. Geromel, and J. Bernussou, "An LMI optimization approach to multiobjective controller design for discrete-time systems," in Decision and Control, 1999. Proceedings of the 38th IEEE Conference on, vol. 4. IEEE, 1999, pp. 3611-3616.

[33] A. White, G. Zhu, and J. Choi, “Optimal LPV control with hard constraints," International Journal of Control, Automation and Systems, vol. 14, no. 1, pp. 148-162, 2016.

\section{BIOGRAPHIES OF AUTHORS}

AQEEL MADHAG (corresponding author)

He received a Ph.D. degree in Electrical and Computer Engineering from Michigan State University, East Lansing, MI 48823, USA in 2019.

Tel.:+964-782-553-4308

E-mail: asr19802007@gmail.com (or madhagaq@msu.edu)

ORCID : 0000-0002-7731-0272

Research ID : N-3948-2018 